\title{
Resolution of Chronic Subdural Hematoma after Treatment with Tumor Necrosis Factor Alpha Inhibitor
}

\author{
Donald Ross \\ Department of Neurological Surgery, OHSU and Portland Veteran's Hospital, Portland, USA. \\ E-mail: rossdo@ohsu.edu
}

Received October $12^{\text {th }}, 2011$; revised November 20 ${ }^{\text {th }}, 2011$; accepted November $29^{\text {th }}, 2011$.

\begin{abstract}
Background and Importance: Chronic subdural hematomas (cSDH) are a common problem for which solutions remain imperfect. Surgery is effective, but not without risk. Recent data have suggested a role for inflammation in the genesis of cSDH and several reports have documented some benefit to steroid treatment. In this report, a possible role for tumor necrosis factor alpha blockade in the resolution of a multiply recurrent cSDH is described. Clinical Presentation: An 86-year-old man with rheumatoid arthritis treated with infliximab presented with a large, symptomatic, multiloculated cSDH. Infliximab was withheld and craniotomy for evacuation was uncomplicated, but recurrent symptoms were noted and a recurrence was operated upon again several weeks later. Follow up CT showed a second recurrence. The patient requested to go back on his infliximab due to painful arthralgias. After a single dose of $10 \mathrm{mg} / \mathrm{kg}$, follow up $C T$ showed that the $C S D H$ resolved and did not recur. Conclusion: Anti-TNF-alpha treatment with infliximab may have played a role in the resolution of this patient's cSDH. Further investigation of this possible effect seems warranted.
\end{abstract}

Keywords: Chronic Subdural Hematoma, Tumor Necrosis Factor Alpha, Inflammation

\section{Introduction}

Symptomatic chronic subdural hematomas (cSDH) have been successfully treated surgically for many years (see Lega et al., 2010 for review) [1]. Recent evidence suggests that inflammation may be critical to the genesis of cSDH, [2] which has lead to reports of successful treatment with steroids. This report describes a patient with a multiply recurrent $\mathrm{CSDH}$, which resolved when the patient was treated with a tumor necrosis factor (TNF)alpha inhibitor for pre-existing rheumatoid arthritis. This suggests that TNF-alpha may play a role in the development of cSDH.

\section{Case Report}

An 86-year-old man with rheumatoid arthritis treated with infliximab (Remicade, [3] Centocor, Malvern, PA) and methotrexate fell striking his head on a chair several months prior to presentation with headache, memory loss, and gait disturbance. Computed tomography (CT) revealed a large, left-sided, multiloculated cSDH (Figure 1). Prophylactic levatiracetam at a dose of $500 \mathrm{mg}$ b.i.d.
(UCB S.A., Brussels, Belgium) was started. Methotrexate and TNF-alpha inhibitor treatment were withheld to avoid wound healing difficulty. The patient underwent a craniotomy for drainage and removal of the loculated membranes. A subdural drain was left in place for 36 hours, during which time the patient was kept flat in bed, and a tapering dose of dexamethasone (4 $\mathrm{mg}$ b.i.d. to $0 \mathrm{mg}$ ) administered for 10 days. A postoperative day one CT scan showed marked reduction in the subdural collection but incomplete re-expansion of the brain, with a persisting $13 \mathrm{~mm}$ subdural fluid collection. The patient improved neurologically and was discharged to home. When the patient developed recurrence of his presenting symptoms 4 weeks later, a follow up CT revealed persistence of the subdural collection, but no interval enlargement. Laboratory work indicated thrombocytopenia with a platelet count of 68,000. Levatiracetam was withheld and the platelet count returned to normal. Symptoms persisted and a subdural drain was reinserted via one of the previous burr holes and the patient was again kept flat in bed for 24 hours and given another tapering dose of dexamethasone for a further 10 days. Postoperative day 


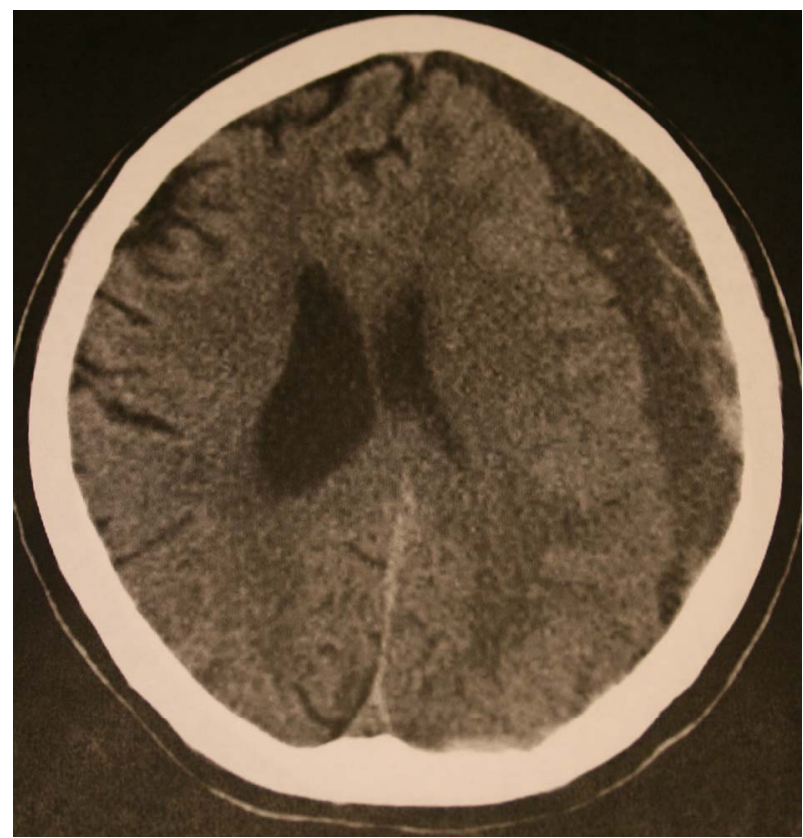

Figure 1. Unenhanced axial CT scan showing a left, multiloculated chronic subdural hematoma at time of presentation.

one CT showed good resolution of the subdural collection with re-expansion of the brain. The patient's symptoms again resolved, but a follow up CT 4 weeks later (8 weeks after the first craniotomy) showed that the collection had begun to reaccumulate (Figure 2). Discussion of treatment options with the patient and his family lead to a decision to not immediately undertake a further operation. The patient's rheumatoid arthritis was becoming problematic for the patient and he requested resumption of his TNF-alpha inhibition treatment. His rheumatologist administered an intravenous dose of Remicade $(10 \mathrm{mg} / \mathrm{kg}$ ). Four weeks later (3 months after his first craniotomy), a follow up CT revealed near complete resolution of the cSDH (Figure 3). The cSDH has not recurred and the patient remains asymptomatic to date, more than one year postoperatively.

\section{Discussion}

In 1998, Suzuki et al., [2] reported that interleukin (IL)-6 and IL-8, but not TNF-alpha or IL-1beta, were elevated in $\mathrm{CSDH}$ specimens from 34 patients. Elevations were not found in plasma or in fluid from 9 subdural effusions, suggesting that inflammation may be involved in the pathogenesis of cSDH. Others have advanced this same hypothesis [4].

In 2001, Shono et al., [5] reported that vascular endothelial growth factor (VEGF), but not basic fibroblast growth factor (bFGF), was elevated in cSDH fluid and that VEGF mRNA was expressed in macrophages and

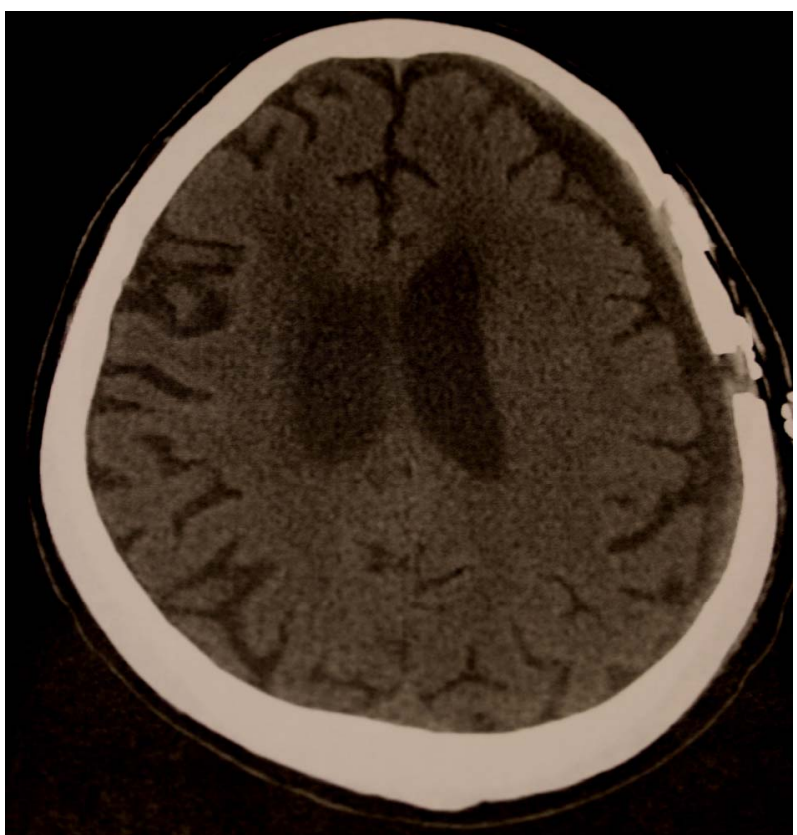

Figure 2. Unenhanced axial CT scan (8 weeks post initial craniotomy) showing recurrent chronic subdural hematoma after two drainage procedures and two courses of steroids.

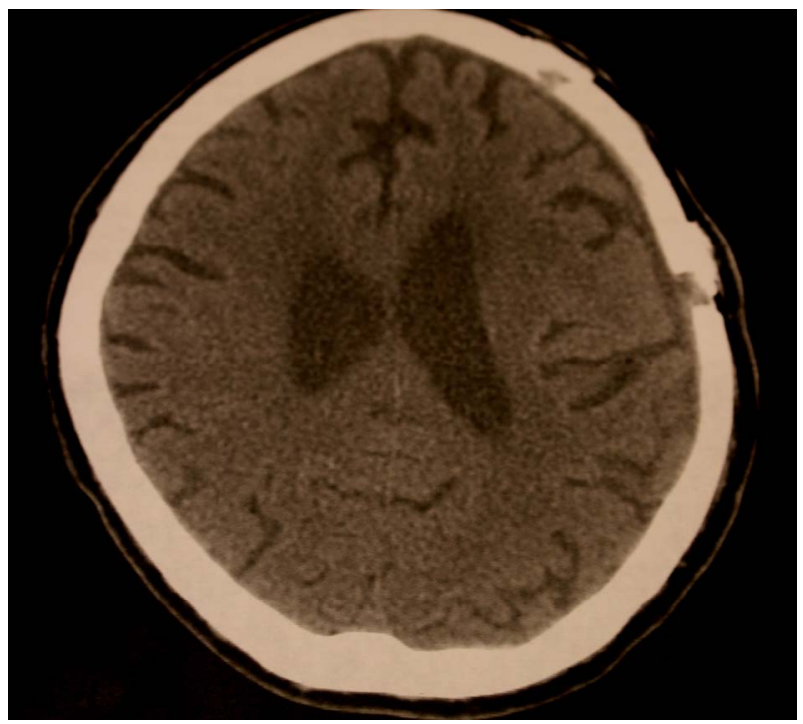

Figure 3. Unenhanced axial CT scan (3 months post initial craniotomy) showing significant resolution of recurrent chronic subdural hematoma after a single infusion of infliximab.

vascular endothelial cells in the outer membranes of cSDHs. These authors concluded that VEGF may be involved in the pathogenesis of cSDH. Others have reported similar findings [6]. Higher levels of IL-6 in cSDH fluid and higher levels of VEGF and bFGF in the membranes have been reported in recurrent cSDHs when compared with nonrecurrent collections [7]. Katano, et al. 
[8] also reported that angiogenic factors, such as hepatocyte growth factor (HGF) and VEGF, may be markers of cSDH enlargement and reported that increased levels of tissue plasminogen activator (TPA) may be indicative of a high probability of recurrence. VEGF is thought to regulate angiogenesis through the phosphatidylinositol 3kinase/Akt pathway; therefore, examination of the outer membranes of cSDH from surgical specimens has revealed that P13, Akt, eNOS, and VE-cadherin were detected in the endothelial cells in all cases [9]. Akt, VEGF, and VE-cadherin were localized to the endothelial cells of vessels within the membranes. Abnormally permeable capillaries in the outer membranes of cSDHs have been thought to be responsible for exudation into the fluid collection, especially in patients with more severe disease [10].

Subsequent to the initial reports of inflammatory processes in cSDHs, reports of successful treatment of cSDHs with dexamethasone have been published [11-13]. In one recent series, $78.2 \%$ of 101 patients treated with dexamethasone avoided surgery [13]. Noting the antiangiogenic effect of angiotensin converting enzyme (ACE) inhibition, Weigel, et al. [14] reported lower VEGF levels in the cSDH fluid and a lower recurrence rate in patients with $\mathrm{CSDH}$ who were taking an ACE inhibitor at the time of treatment.

Activities attributed to TNF-alpha include, induction of pro-inflammatory cytokines IL-1 and IL-6 (the latter noted above to be elevated in cSDH fluid), enhancement of leukocyte migration, activation of neutrophil function, induction of acute phase reactants, and activation of tissue degrading enzymes [3]. TNF-alpha has been implicated in the blood vessel remodeling and lymphangiogenesis observed in inflammation [15]. Infliximab (Remicade [3]) is a chimeric monoclonal antibody which neutralizes the biological activity of TNF-alpha by binding to it with high affinity and inhibiting binding with its receptors $[16,17]$. Infliximab is indicated in the treatment of rheumatoid arthritis, Crohn's disease, ankylosing spondylitis, psoriasis and psoriatic arthritis, and ulcerative colitis.

\section{Conclusions}

Although it cannot be shown with certainty that this single patient's CSDH resolved as a result of treatment with infliximab, the fact that the hematoma had recurred twice after drainage and treatment with dexamethasone, but resolved after a single dose of infliximab is at least suggestive that the TNF-alpha inhibitor was responsible for this unexpected result. The reported incidence of cSDH is 1 - 5.3 cases per 100,000 people per year [18] and up to 7.35 cases per 100,000 per year in the 70 - 79 year old age group [19]; therefore, as many as 16,000 cases may occur yearly in the United States. A recent study of 209 patients over 65 years of age reported an in hospital mortality of $16.7 \%$ and a six month mortality of $26.3 \%$, indicating that $\mathrm{cSDH}$ is "not a benign disease" [20]. Study of a larger group of patients seems warranted.

\section{REFERENCES}

[1] B. C. Lega, S. F. Danish, N. R. Malhotra, S. S. Sonnad and S. C. Stein, "Choosing the Best Operation for Chronic Subdural Hematoma: A Decision Analysis," Journal of Neurosurgery, Vol. 113, No. 3, 2010, pp. 615621. doi:10.3171/2009.9.JNS08825

[2] M. Suzuki, S. Endo, K Inada, et al., "Inflammatory Cytokines Locally Elevated in Chronic Subdural Haematoma," Acta Neurochirurgica (Wien), Vol. 140, No. 1, 1998, pp. 51-55. doi:10.1007/s007010050057

[3] “Physicians’ Desk Reference,” Thomson Reuters, Montvale, 2009.

[4] J. F. Feng, J. Y. Jiang, Y. H. Bao, Y. M. Liang and Y. H. Pan, "Traumatic Subdural Effusion Evolves into Chronic Subdural Hematoma: Two Stages of the Same Inflammatory Reaction?” Medical Hypotheses, Vol. 70, No. 6, 2008, pp. 1147-1149. doi:10.1016/j.mehy.2007.11.014

[5] T. Shono, T. Inamura, T. Morioka, et al., "Vascular Endothelial Growth Factor in Chronic Subdural Haematomas,” Journal of Clinical Neuroscience, Vol. 8, No. 5, 2001, pp. 411-415. doi:10.1054/jocn.2000.0951

[6] N. Nanko, M. Tanikawa, M. Mase, et al., "Involvement of Hypoxia-Inducible Factor-1alpha and Vascular Endothelial Growth Factor in the Mechanism of Development of Chronic Subdural Hematoma," Neurologia MedicoChirurgica (Tokyo), Vol. 49, No. 9, 2009, pp. 379-385. doi:10.2176/nmc.49.379

[7] H. J. Hong, Y. J. Kim, H. J. Yi, Y. Ko, S. J. Oh and J. M. Kim, "Role of Angiogenic Growth Factors and Inflammatory Cytokine on Recurrence of Chronic Subdural Hematoma,” Surgical Neurology, Vol. 71, No. 2, 2009, pp. 161-166.

[8] H. Katano, K. Kamiya, M. Mase, M. Tanikawa and K. Yamada, "Tissue Plasminogen Activator in Chronic Subdural Hematomas as a Predictor of Recurrence,” Journal of Neurosurgery, Vol. 104, No. 1, 2006, pp. 79-84. doi:10.3171/jns.2006.104.1.79

[9] M. Funai, K. Osuka, N. Usuda, et al., “Activation of P13 kinase/Akt Signaling in Chronic Subdural Hematoma Outer Membranes,” Journal of Neurotrauma, Vol. 28, No. 6, 2011, pp. 1127-1131. doi:10.1089/neu.2010.1498

[10] M. Tokmak, A. C. Iplikcioglu, S. Bek, C. A. Gokduman and M. Erdal, "The Role of Exudation in Chronic Subdural Hematomas,” Journal of Neurosurgery, Vol. 107, No. 2, 2007, pp. 290-295. doi:10.3171/JNS-07/08/0290

[11] O. Decaux, B. Cador, T. Dufour, et al., "Traitement des Hematomes Sous-Duraux Chroniques par les Corticoides: A Propos de Deux Observations,” La Revue de Médecine Interne, Vol. 23, No. 9, 2002, pp. 788-791. doi:10.1016/S0248-8663(02)00676-8 
[12] T. F. Sun, R. Boet and W. S. Poon, "Non-Surgical Primary Treatment of Chronic Subdural Haematoma: Preliminary Results of Using Dexamethasone," British Journal of Neurosurgery, Vol. 19, No. 4, 2005, pp. 327333. doi:10.1080/02688690500305332

[13] P. D. Delgado-Lopez, V. Martin-Velasco, J. M. CastillaDiez, A. Rodriguez-Salazar, A. M. Galacho-Harriero and O. Fernandez-Arconada, "Dexamethasone Treatment in Chronic Subdural Haematoma,” Neurocirugia (Astur), Vol. 20, No. 4, 2009, pp. 346-359. doi:10.4321/S1130-14732009000400003

[14] R. Weigel, A. Hohenstein, L. Schlickum, C. Weiss and L. Schilling, “Angiotensin Converting Enzyme Inhibition for Arterial Hypertension Reduces the Risk of Recurrence in Patients with Chronic Subdural Hematoma Possibly by an Antiangiogenic Mechanism,” Neurosurgery, Vol. 61, 2007, pp. 788-783.

[15] P. Baluk, L. C. Yao, J. Feng, et al., “TNF-Alpha Drives Remodeling of Blood Vessels and Lymphatics in Sustained Airway Inflammation in Mice," The Journal of Clinical Invest, Vol. 119, 2009, pp. 2954-2964.

[16] D. M. Knight, H. Trinh, J. Le, et al., "Construction and
Initial Characterization of a Mouse-Human Chimeric Anti-TNF Antibody," Molecular Immunology, Vol. 30, No. 16, 1993, pp. 1443-1453. doi:10.1016/0161-5890(93)90106-L

[17] B. J. Scallon, M. A. Moore, H. Trinh, D. M. Knight and J. Ghrayeb, "Chimeric Anti-TNF-Alpha Monoclonal Antibody cA2 Binds Recombinant Transmembrane TNF-Alpha and Activates Immune Effector Functions,” Cytokine, Vol. 7, No. 3, 1995, pp. 251-259. doi:10.1006/cyto.1995.0029

[18] H. Engelhard III, G. Sinson and T. Reiter, "Subdural Hematoma," eMedicine Neurosurgery, 2009, Accessed on 2 February 2011.

http://emedicine.medscape.com/article/247472-followup

[19] R. Meagher and W. Young, "Subdural Hematoma," eMedicine Neurology, 2009, Accessed on 2 February 2011.

http://emedicine.medscape.com/article/1137207-overview

[20] L. B. Miranda, E. Braxton, J. Hobbs and M. R. Quigley, "Chronic Subdural Hematoma in the Elderly: Not a Benign Disease,” Journal of Neurosurgery, Vol. 114, No. 1, 2011, pp. 72-76. doi:10.3171/2010.8.JNS10298 